\title{
Brain activity during biofeedback relaxation A functional neuroimaging investigation
}

\author{
H. D. Critchley, ${ }^{1,2}$ R. N. Melmed, ${ }^{1,5}$ E. Featherstone, ${ }^{1}$ C. J. Mathias ${ }^{2,3}$ and R. J. Dolan ${ }^{1,4}$ \\ ${ }^{1}$ Wellcome Department of Cognitive Neurology, Institute of \\ Neurology, UCL, ${ }^{2}$ Autonomic Unit, National Hospital for \\ Neurology and Neurosurgery and Institute of Neurology, \\ UCL, ${ }^{3}$ Department of Neurovascular Medicine, St Mary's \\ Correspondence to: H. D. Critchley, Wellcome Department \\ of Cognitive Neurology, 12 Queen Square, ION, UCL, \\ London WC1N 3BG, UK \\ E-mail: h.critchley@fil.ion.ucl.uk
} Hospital, Imperial College School of Medicine, ${ }^{4}$ Royal Free

Hospital, University College Hospital School of Medicine, UCL, London, UK and ${ }^{5}$ Department of Medicine, Hadassah University Hospital, Jerusalem, Israel

\section{Summary}

The mechanisms by which cognitive processes influence states of bodily arousal are important for understanding the pathogenesis and maintenance of stress-related morbidity. We used PET to investigate cerebral activity relating to the cognitively driven modulation of sympathetic activity. Subjects were trained to perform a biofeedback relaxation exercise that reflected electrodermal activity and were subsequently scanned performing repetitions of four tasks: biofeedback relaxation, relaxation without biofeedback and two corresponding control conditions in which the subjects were instructed not to relax. Relaxation was associated with significant increases in left anterior cingulate and

globus pallidus activity, whereas no significant increases in activity were associated with biofeedback compared with random feedback. The interaction between biofeedback and relaxation, highlighting activity unique to biofeedback relaxation, was associated with enhanced anterior cingulate and cerebellar vermal activity. These data implicate the anterior cingulate cortex in the intentional modulation of bodily arousal and suggest a functional neuroanatomy of how cognitive states are integrated with bodily responses. The findings have potential implications for a mechanistic account of how therapeutic interventions, such as relaxation training in stress-related disorders, mediate their effects.

Keywords: autonomic; biofeedback; relaxation; positron emission tomography; electrodermal activity

Abbreviation: EDA = electrodermal activity

\section{Introduction}

Electrodermal activity (EDA) results from sympathetic stimulation of eccrine sweat glands in the skin, leading to alteration in the conductance of an applied current. EDA reflects both a relatively rapid transient event, called the skin conductance response, and a slower, gradual shift in the basal level, the skin conductance level. Sympathetic activity is linked to emotional and cognitive states and EDA is widely used as a sensitive index of bodily arousal related to emotion and attention (Venables and Christie, 1980; Fowles et al., 1981; Boucsein, 1992; Dawson et al., 2000). There is also evidence demonstrating that sympathetic arousal, reflected in EDA, can feed back to influence emotional states and cognitive processes such as decision-making and memory (Damasio et al., 1991; Damasio, 1994; Bechara et al., 1996, 1997; Cahill, 1997).

The role of emotion-related autonomic activity in the maintenance and pathophysiological consequences of anxiety and stress disorders is of considerable clinical importance. Chronic high levels of sympathetic arousal are hallmarks of anxiety disorders and stress, conditions associated with high levels of psychological and physical morbidity (Russek et al., 1990; Steptoe et al., 1999). Interventions that lower autonomic arousal result in a reduction of stress and anxiety and increase feelings of well-being. Among these techniques, biofeedback relaxation allows subjects to influence autonomic activity voluntarily by the provision of real-time feedback (often visual) of an autonomic measure such as EDA. Biofeedback relaxation, in which subjects learn to decrease sympathetic arousal, may also provide a quantitative measure of sympathetic tone.

Traditional studies of sympathetic tone using EDA have focused on the amplitude of responses elicited by emotive 
stimuli or during cognitive processing. Evoked peaks in EDA are susceptible to wide inter- and intra-individual variability, environmental influences such as ambient temperature, dependency on recording methods, time effects and, most notably, habituation of response with repeated stimulation (Boucsein, 1992). Biofeedback relaxation tasks involving EDA overcome many of these problems by providing an objective quantitative measure (in the form of latency, or rate of decreasing sympathetic tone) that is largely independent of differences in the amplitude of evoked responses.

Functional imaging experiments have implicated specific brain areas in the generation and feedback representation of autonomic arousal. These regions include those associated with emotion and attention, such as the anterior cingulate, insula, orbitofrontal cortex and inferior parietal lobe (Fredrikson et al., 1998; Critchley et al., 2000a, b). Studies have also been performed examining brain activity relating to meditation (Lazar et al., 2000) and hypnosis (Rainville et al., 1999), in which the activity accompanying these processes highlights a pivotal role for the anterior cingulate cortex. These studies, however, did not investigate in a systematic way the relationship between regional brain activity, intentional states and physiological measures of autonomic relaxation.

The present study examines the influence of cognitive intent on sympathetic relaxation (i.e. diminishing peripheral sympathetic arousal). We trained healthy subjects in the performance of a biofeedback relaxation exercise and used PET to examine the functional neuroanatomical correlates of decreases in EDA, cognitive activity relating to the intention to relax and the influence of biofeedback on these relaxation processes. The goal of the study was to identify brain mechanisms through which cognitive processing is integrated with changes in autonomic bodily responses.

\section{Methods \\ Subjects}

Eight healthy right-handed male volunteers (mean age 41 years, SD 19) were recruited after medical screening to exclude disorders or medication that might affect brain function or perfusion. Female subjects did not take part in the study because of restrictions related to the Administration of Radioactive Substances Advisory Committee licence. Each subject gave full informed consent (Declaration of Helsinki, 1991) to participation in the study, which was approved by the Joint Ethics Committee of the National Hospital for Neurology and Neurosurgery and the Institute of Neurology. Before scanning, subjects were familiarized with the biofeedback relaxation exercise and practised so that they were able perform the task proficiently and reliably (below).

\section{Experimental design}

Subjects performed three repetitions of four tasks in a $2 \times 2$ factorial experimental design. This factorial design enabled examination of regional activity relating to the main effect of relaxation, the main effect of receiving feedback of EDA and the interaction between these factors, which allowed identification of regions where relaxation-related activity was modulated by biofeedback. For each subject, tasks were presented in a unique pseudorandom order. Electrodermal activity was monitored continuously throughout the experiment in all tasks. The biofeedback signal of relaxation was a red, easy-to-see picture of a simple representation of a thermometer (a column and a bulb) on a light grey background, $12 \mathrm{~cm}$ high and $1 \mathrm{~cm}$ in width, viewed on a monitor $1 \mathrm{~m}$ from the subject's head. The thermometer appearing on the screen was a signal to the subject to begin a particular exercise or task. As the subject relaxed, the thermometer column became shorter until all that was left was the thermometer bulb. The change in thermometer column height represented a logarithmic function of changes in the subject's EDA. The column height could also be set to fluctuate randomly (Fig. 1).

\section{Training in the biofeedback relaxation exercise}

Subjects were trained to perform biofeedback relaxation proficiently on the day before scanning. Subjects were briefed about biofeedback techniques and provided with a demonstration of biofeedback relaxation. For a period of $5 \mathrm{~min}$, subjects viewed the display relating to their fluctuating level of sympathetic tone. Subsequently, they were encouraged to try to relax while monitoring the feedback and during the ensuing 30-45 min they learned to modulate their sympathetic tone using biofeedback. Just before scanning, subjects were tested to ensure that they had retained competence in performing the biofeedback relaxation exercise. During scanning, performance of the biofeedback relaxation task was facilitated by gentle physiotherapy between scans, subdued lighting and minimal encouragement of the subjects to perform the task rapidly or correctly. Before performance of each task, subjects were informed fully about whether feedback would reflect their level of arousal or fluctuate randomly and whether they were required to relax during the task.

\section{Experimental tasks}

\section{Biofeedback with intended relaxation}

The subject attempted, through relaxation, to bring the thermometer column down to the bowl. The subjects usually achieved this within 2-3 min, enabling a 90 s PET acquisition to occur during the biofeedback relaxation.

\section{Biofeedback with no relaxation}

The subject was required to watch the display and not relax, but to try to prevent any downward drift of the thermometer 
(A)

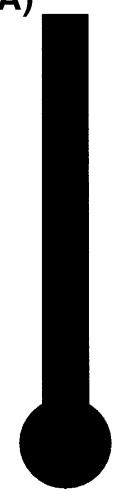

(B)

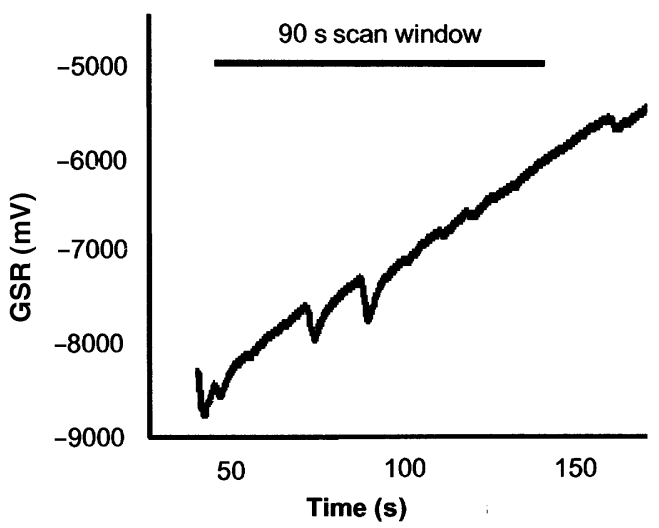

(C)

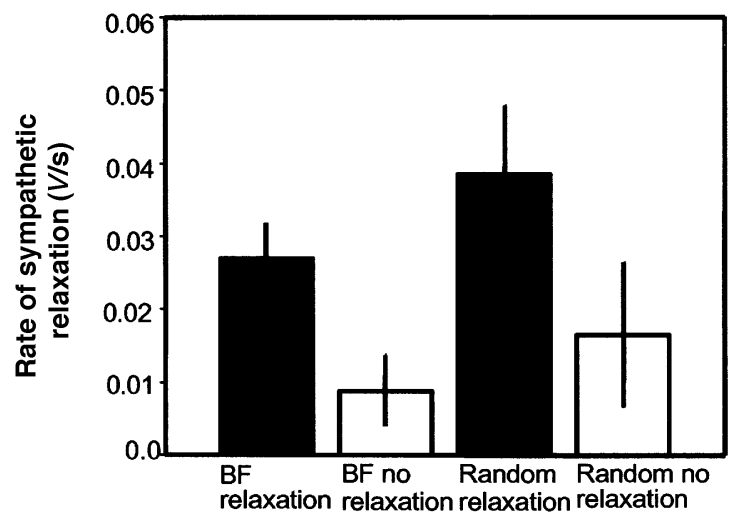

Fig. 1 Visual display and task performance. (A) Thermometer display viewed by subjects in all four experimental tasks. During biofeedback tasks, changes in the height of the thermometer represented a logarithmic function of recorded sympathetic skin response; decreases in bar height towards the bowl corresponded to sympathetic relaxation. In non-biofeedback tasks, bar height fluctuated randomly with a temporal frequency approximating the rate of change in bar height during the biofeedback relaxation task. (B) Example time-course of sympathetic relaxation, measured as the galvanic skin response (GSR), during scanning. The subject was prompted to begin relaxation at time zero. Scanning data acquisition began at $60 \mathrm{~s}$ and lasted $90 \mathrm{~s}$. The derived voltage across the skin increased towards zero as the subject decreased sympathetic tone. (C) Rates of relaxation across all subjects during performance of the four experimental tasks. $\mathrm{BF}=$ biofeedback given in thermometer display; random = no biofeedback (random fluctuations) in the thermometer display. The figure shows that greater sympathetic relaxation was present in relaxation tasks than in control tasks and that during scanning there were higher rates of relaxation when no biofeedback was given during relaxation, which is attributable in part to performance anxiety in trained subjects.

column by any strategy that did not involve movement of the limbs or body.

\section{Random feedback with intended relaxation}

The subject viewed the same video display of a thermometer that fluctuated randomly at the upper limit of the column height. While watching the display, the subject was required to relax, although the display was unrelated to the subject's physiological responses.

\section{Random feedback with no relaxation exercise}

As in the previous task, subjects were required to watch a video display fluctuating randomly, which did not reflect their level of arousal. During this experimental task, subjects were told to attempt not to relax, as in the biofeedback with no relaxation task.

\section{Monitoring of EDA and biofeedback}

EDA, reflecting predominantly skin conductance level, was monitored continuously with silver electrodes taped to the palmar surface of the left index and middle fingers and the signal was fed via a skin conductance processing unit (SCL 200; Biofeedback Systems, Manchester, UK). The filtered analogue output of EDA was displayed on-line and recorded digitally on a PC into MATLAB (MathWorks, Sherborn, Mass., USA). Within MATLAB, a feedback function was created for the purpose of providing visual display to the subject during biofeedback exercises. The raw EDA data (smoothed over $25 \mathrm{~ms}$ then sampled every $100 \mathrm{~ms}$ ) was transformed by a natural logarithm and scaled. At the start of the biofeedback tasks, the level of this function was normalized to 1 and the thermometer display reflected the height of this normalized function (Fig. 1). In trials in which no biofeedback was given, the thermometer display was driven by a random function [new value $=$ random number/ 10 - (old value -0.5$) / 5+$ old value], biased towards the mean of the display, which was then smoothed temporally to mimic the time-course of physiological fluctuations. Data relating to the height of the visual feedback and raw level of EDA were recorded for each scan. Post hoc processing of the EDA data determined the mean gradient of changes in raw EDA over the course of each $90 \mathrm{~s}$ data acquisition period. The rates of relaxation per scan were entered into analysis as two covariates, corresponding to (i) rates of decremental change when performing (biofeedback and nonbiofeedback) relaxation tasks and (ii) rates of decremental change during the performance of non-relaxation tasks.

\section{PET scan acquisition and analysis}

Scans of the distribution of $\mathrm{H}_{2}{ }^{15} \mathrm{O}$ were obtained with a Siemens/CPS ECAT EXACT HR + PET Scanner operated in high-sensitivity $3 \mathrm{D}$ mode. Subjects received a total of $350 \mathrm{Mbq}$ of $\mathrm{H}_{2}{ }^{15} \mathrm{O}$ over $20 \mathrm{~s}$ through a right antecubital cannula for each of the 12 scans and activity was measured 
Table 1 Experimental design

\begin{tabular}{lll}
\hline & \multicolumn{2}{l}{ Feedback presented to subject } \\
\cline { 2 - 3 } & Biofeedback of EDA & $\begin{array}{l}\text { Random fluctuation } \\
\text { (no biofeedback) }\end{array}$ \\
\hline Intention to relax & Task A, 3 scans & $\begin{array}{l}\text { Task C, 3 scans } \\
\text { Task D, 3 scans }\end{array}$ \\
\hline Intention not to relax & Task B, 3 scans & Ras \\
\hline
\end{tabular}

during a $90 \mathrm{~s}$ time window while the subjects performed the experimental tasks. The PET images comprised $i, j$ and $k$ voxels $(2 \times 2 \times 3 \mathrm{~mm})$ with $6.4 \mathrm{~mm}$ transaxial and $5.7 \mathrm{~mm}$ axial resolution (full width at half maximum). The data were analysed by statistical parametric mapping (SPM99; Wellcome Department of Cognitive Neurology, London, UK) implemented in MATLAB. Structural MRIs from each subject were co-registered to the PET data after realignment of the PET time series. All the scans were then transformed into a standard stereotactic space (Talairach and Tournoux, 1988; Friston et al., 1995b). The scans were smoothed using a Gaussian filter set at $10 \mathrm{~mm}$ full width at half maximum. These regional cerebral blood flow measurements were adjusted to a global mean of $50 \mathrm{ml} / \mathrm{dl}$ per min.

Data were analysed after construction of a design matrix for the analysis of group data for conditions and covariates. Global CBF was entered in the design matrix as ANCOVA (analysis of covariance) by subject and treated as a confounding covariate. The rate of sympathetic relaxation for each scan was entered into the analytical design as a regressor to permit exploration of interactions between task condition and measured sympathetic relaxation. Our analyses used a factorial design to explore the main effects of relaxation, biofeedback and relaxation $\times$ biofeedback interaction (Table 1). This approach allowed the identification of regional brain activity related to relaxation (independent of visual feedback), activity related to biofeedback of EDA (independent of intended relaxation) and regions where relaxation-related activity was modulated by biofeedback. Additionally, this factorial approach had greater statistical power than reporting simple effects, as a consequence of using all the collected data. Analyses of the rate of measured relaxation were also undertaken to determine brain areas associated with changes in sympathetic tone common to relaxation and non-relaxation tasks. Lastly, relationships between measured relaxation rates and the activity of brain regions identified in earlier analyses were explored post hoc using Pearson correlations. The general methods employed by statistical parametric mapping have been described in detail by Friston and colleagues (Friston et al., 1995a,b).

On the basis of observations about the cerebral control of autonomic function from lesion, stimulation and functional imaging studies in humans, we predicted that activity in specific brain regions would be associated with sympathetic relaxation and performance of the biofeedback relaxation exercise. Lesions of the anterior cingulate, orbitofrontal cortex, insula, amygdala and inferior parietal lobe are known to affect EDA measures of sympathetic arousal (Damasio et al., 1991; Tranel and Damasio, 1994; Bechara et al., 1995). Stimulation of anterior cingulate, insula and amygdala/medial temporal lobe modulate cardiovascular and visceral autonomic responses (Pool and Ransohoff, 1949; Oppenheimer et al., 1992; Fish et al., 1993). Functional imaging studies have reported an association between the anterior cingulate, orbital, ventromedial prefrontal, insula and inferior parietal cortices, amygdala, pons and cerebellar vermis with cardiovascular or EDA measures of autonomic arousal (Williamson et al., 1997; Buchel et al., 1998; Fredrikson et al., 1998; Soufer et al., 1998; Critchley et al., $2000 a, b)$. We thresholded our analyses initially at $P<0.001$ uncorrected, and subsequently report activity within voxel clusters exceeding 10 voxels, where peak activation reached significance corrected for whole brain at $P<0.05$. Additionally, to avoid type 2 errors for regions predicted a priori, we used small volume corrections (Worsley et al., 1996). If activity in brain regions previously implicated in autonomic control, i.e. the anterior cingulate, orbitofrontal, insula, and inferior parietal cortices, amygdala, pons and cerebellar vermis, or their contralateral homologues, reached significance at $P<0.001$ uncorrected, then we tested for corrected significance using spherical small volumes of radius $10 \mathrm{~mm}$ centred on coordinates derived from previous independent functional imaging experiments (Worsley et al., 1996). Thus, we used the following $x, y, z$ coordinates (lateral, anterior-posterior and vertical distances in millimetres) for small volume corrections: anterior cingulate, $\pm 16,20,38$ (Critchley et al., 2000a); medial prefrontal cortex, $\pm 14,46,2$ (Critchley et al., 2000b); orbitofrontal cortex/anterior insula, $\pm 36,26,-12$ (Critchley et al., 2000b); insula, $\pm 58,-6,14$ (Critchley et al., 2000a); inferior parietal cortex, $\pm 42,-34$, 24 (Critchley et al., 2000b); amygdala, 土24, 3, -24 (Buchel et al., 1998; Critchley et al., 2000a); pons, $\pm 16,-30,-42$ (Critchley et al., 2000a); and cerebellar vermis, $\pm 2,-52$, -20 (Critchley et al., 2000a). Talairach coordinates are reported (Talairach and Tournoux, 1988) and the atlas of Duvernoy (Duvernoy, 1991) was used as a reference for the identification of neuroanatomical locations.

\section{Results \\ Behavioural performance and debriefing}

At debriefing, subjects described using two principal methods of engendering sympathetic relaxation: reduction of limb tension and movement (in any case, a necessity during scanning) and making slower, shallower breaths. Visualization of pleasant scenes or colours was also employed by some of the subjects. When relaxation was achieved effectively, the subjects' reports were consistent with increased somnolence and sense of well-being. Despite adequate pretraining, variability within and between subjects was noted in the performance of the biofeedback relaxation 


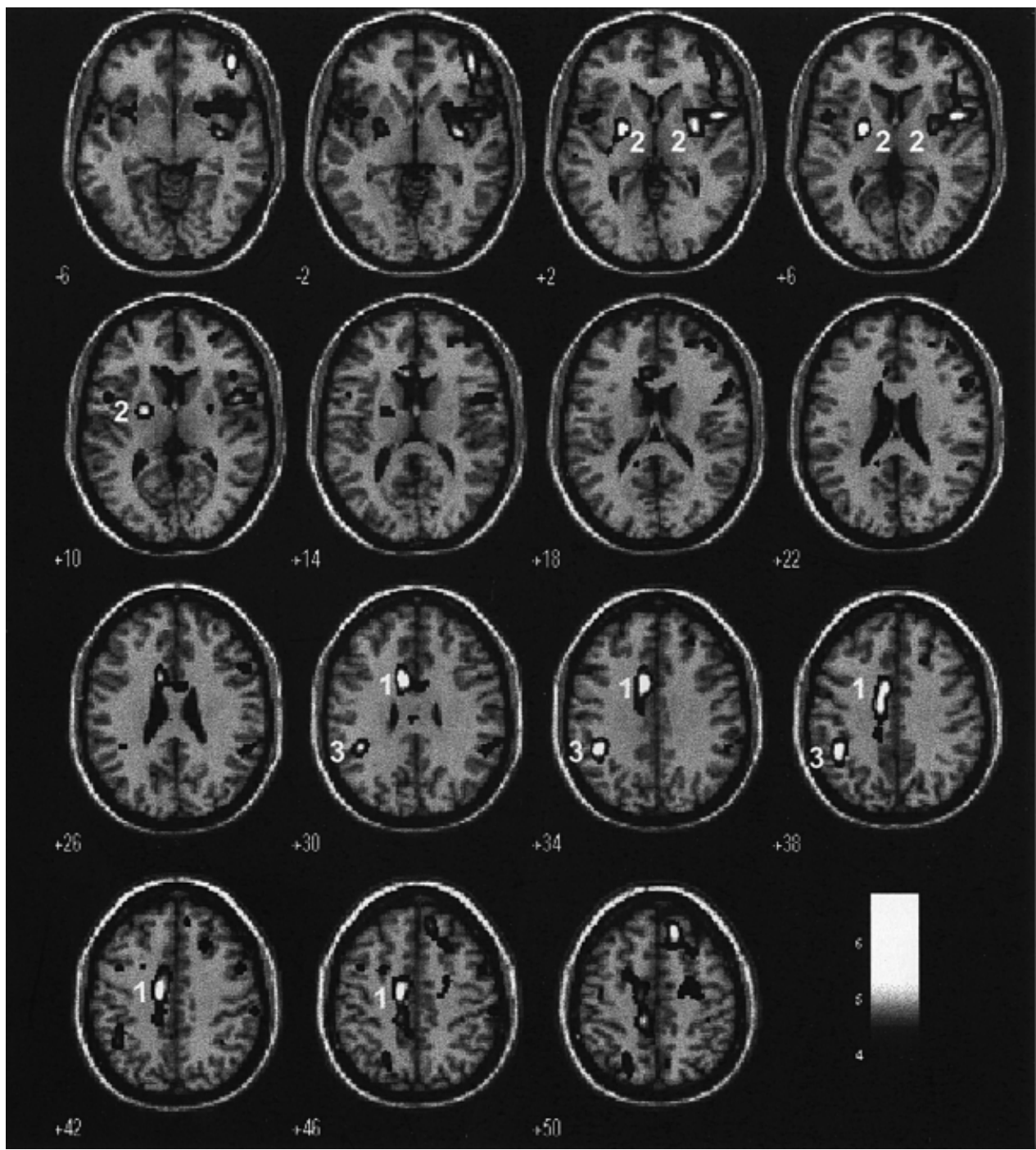

KEY

\section{1 anterior} cingulate

2 globus pallidus

3 inferior parietal lobule

Fig. 2 Activity related to the main effect of performing relaxation tasks. Group data ( $P>0.001$, uncorrected, for illustrative purposes) is illustrated on 15 axial sections of a normalized template image derived from one subject. Height in millimetres $(z$ coordinate) above the anterior commissure is given adjacent to each section. The graded bar illustrates the $Z$ scores of the activations. Locations of activation are indicated in the numbered key. Greatest activity related to sympathetic relaxation was observed in the left anterior cingulate and globus pallidus $(P<0.05$, corrected).

exercise, consistent with a degree of performance anxiety at the time of scanning. Nevertheless, across the group, biofeedback and non-biofeedback relaxation tasks elicited decreases in EDA that produced significant between-task differences in the rate of sympathetic relaxation $[F(3)=$ 2.9, $P<0.05$ ) (Fig. 1).

\section{Brain activity related to task performance}

The main effect of relaxation on brain activity was assessed by comparing relaxation with non-relaxation tasks (with and without biofeedback). Increases in activity were observed in a discrete set of brain regions, including the left anterior cingulate and globus pallidus (both significant at $P<0.05$, corrected) associated with intentional relaxation. Enhanced relaxation-related activity was also observed in the left inferior parietal lobule $(P<0.05$, corrected for small volume $)$ (Table 2 and Fig. 2). The main effect of biofeedback (compared with presentation of random fluctuations of the feedback thermometer) did not reveal any regions reaching corrected significance.

\section{Modulation of relaxation as a function of feedback}

By examining the interaction between biofeedback and relaxation tasks, we sought to identify regions where relaxation-related activity was modulated by biofeedback. The comparison of activity during relaxation versus nonrelaxation in the presence versus absence of biofeedback revealed significant activity in the cerebellar vermis and 
bilateral anterior cingulate/medial prefrontal cortex $(P<0.05$, corrected for small volume) (Table 2 and Fig. 3).

\section{Activity relating to measured rate of relaxation}

We next examined brain activity related to the rate of change in EDA during each scan. These rates formed two regressors within the design matrix corresponding to relaxation rates: (i) when the subjects were attempting to decrease their sympathetic tone; and (ii) when the subjects were actively not attempting such relaxation. Across both relaxation and non-relaxation tasks and during relaxation tasks alone, activity covarying with EDA was observed adjacent to the right amygdala $(x, y, z$ coordinates $18,10,-28$ and 18,0 , $-28 ; P<0.05$, corrected for amygdala volume) (Table 3 and Fig. 4).

\section{Activity relating to intended and successful relaxation}

Finally, we examined how activity correlated with measured rates of change in EDA, in regions expressing a significant

Table 2 Activity related to task performance

\begin{tabular}{|c|c|c|c|c|c|c|}
\hline \multirow[t]{2}{*}{ Area } & \multirow{2}{*}{$\begin{array}{l}\text { Brodmann } \\
\text { area }\end{array}$} & \multirow[t]{2}{*}{ Side } & \multicolumn{3}{|c|}{ Coordinates } & \multirow[t]{2}{*}{$Z$ score } \\
\hline & & & $z$ & $y$ & $z$ & \\
\hline \multicolumn{7}{|c|}{ Activity relating to performance of relaxation tasks } \\
\hline Anterior cingulate & 24 & Left & -8 & 6 & 32 & $4.77 *$ \\
\hline Globus pallidus & - & Left & -24 & -4 & 4 & $4.73 *$ \\
\hline Inferior parietal lobule & 23 & Left & -42 & -42 & 30 & $3.59^{\dagger}$ \\
\hline \multicolumn{7}{|c|}{ Biofeedback $\times$ relaxation interaction } \\
\hline Cerebellar vermis & - & Left & -6 & -56 & -12 & $3.74^{\dagger}$ \\
\hline $\begin{array}{l}\text { Anterior cingulate/ } \\
\text { ventromedial }\end{array}$ & 24 & Right & t 12 & 42 & 10 & $3.42^{\dagger}$ \\
\hline Prefrontal cortex & & & & & & \\
\hline Anterior cingulate & $24 / 32$ & Left & -16 & 20 & 28 & $3.21^{\dagger}$ \\
\hline
\end{tabular}

Significant at $P<0.05$ corrected $*$ for whole brain and ${ }^{\dagger}$ for small volume for predicted region of interest. main effect of intentional relaxation. As described above, activity in the left anterior cingulate and globus pallidus discriminated strongly between relaxation and non-relaxation tasks. We found that activity in the anterior cingulate correlated negatively with the rate of EDA change during relaxation tasks (Pearson $r=-0.81, P<0.001$ ) but not during non-relaxation tasks ( $r=0.27$, not significant). Thus, activity in the anterior cingulate reflected the intention to relax, activity being highest when sympathetic tone was not decreasing. Activity in the globus pallidus, in contrast to the anterior cingulate, correlated significantly with the rate of EDA change during both relaxation (negatively, $r=0.67$, $P<0.001$ ) and non-relaxation tasks (positively, $r=0.49$, $P<0.001)$. Thus, activity in the globus pallidus was increased during the intention to relax and also increased when these intentions were not realized in the relaxation or non-relaxation tasks.

\section{Discussion}

A knowledge of the mechanisms through which cognitive processing influences bodily responses is important for understanding stress-related morbidity, psychosomatic illness and the mechanistic basis of therapeutic interventions in cognitive and behavioural therapies. We know little, however, about how cognitive processing influences bodily responses, despite the fact that such interactions are central to an influential theory of emotion and decision-making (Damasio et al., 1991; Damasio, 1994; Bechara et al., 1997). Previous studies have described brain activity associated with the generation and feedback representation of states of bodily arousal (e.g. Fredrikson et al., 1998; Soufer et al., 1998; Critchley et al., 2000a, b) and also activity evoked by 'cognitive relaxation' techniques, such as meditation and hypnotic induction (Rainville et al., 1999; Lazar et al., 2000). This study, however, is the first to address systematically the question of brain activity in relation to a subject's intention to relax, both with and without the aid of biofeedback.

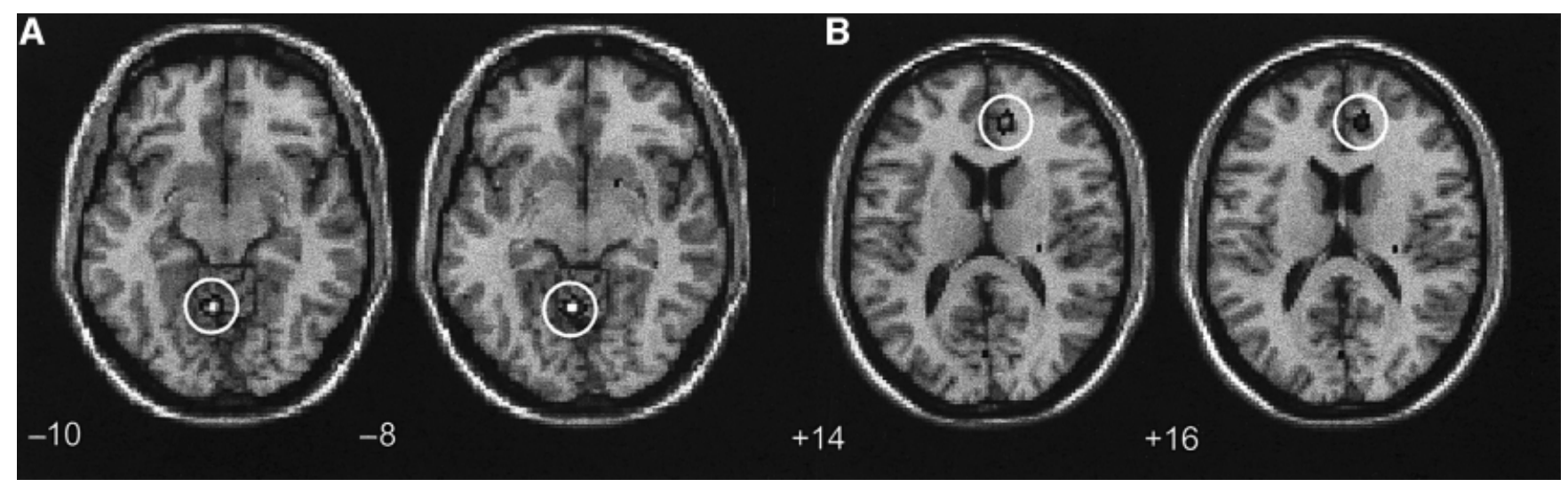

Fig. 3 Activity related to biofeedback relaxation. Significant $(P<0.05$ corrected for small volumes) activity related to biofeedback $\times$ relaxation interaction. This analysis identifies activity greater in biofeedback relaxation (versus the biofeedback control task) than in relaxation without biofeedback (versus the non-biofeedback control task). Group data are plotted on four axial sections of a template structural scan illustrating (A) activity in the cerebellar vermis and (B) activity in the right anterior cingulate. Height (in mm) ( $z$ coordinate) above the anterior commissure is given below each section. 
(A)

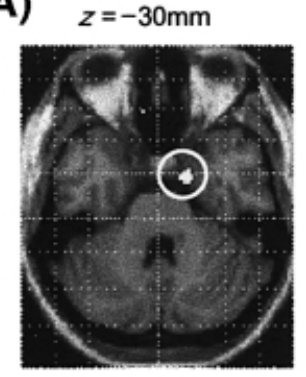

$z=-28 \mathrm{~mm}$

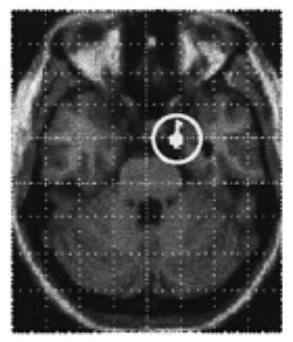

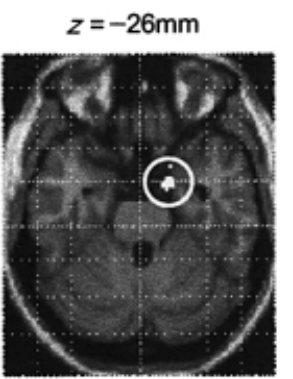

(B)

$$
\begin{aligned}
& \text { Right periamygdala activity related to } \\
& \text { rate of sympathetic relaxation }
\end{aligned}
$$

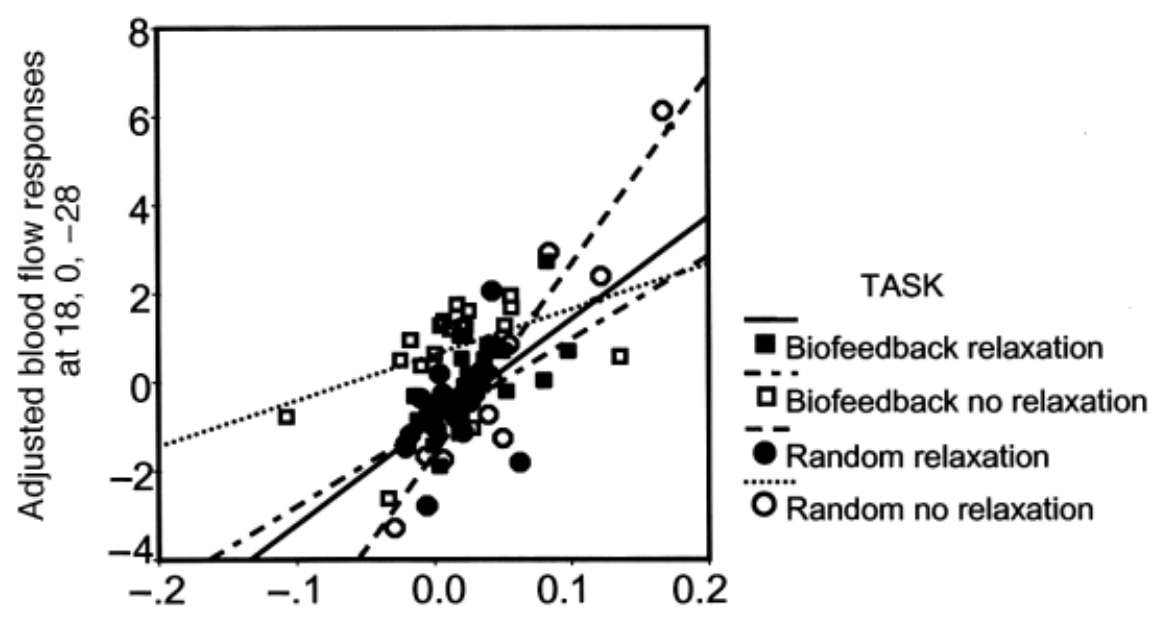

Rate of decreases in skin conductance (V/s)

Fig. 4 Activity related to the measured rate of relaxation. (A) Activity relating to the rate of sympathetic relaxation during relaxation tasks, in the right anterior medial temporal lobe, located anterior and inferior to the right amygdala (significant at $P<0.05$, corrected for a small volume centred on the amygdala coordinates). Group data are plotted on axial sections of a template image; height in millimetres is given above each section. (B) Plot of adjusted changes in cerebral blood flow in the periamygdala region against the measured rates of sympathetic relaxation in all four experimental tasks. Regression lines are plotted for each task. In this periamygdala region there was a significant positive correlation between the rate of sympathetic relaxation and the adjusted blood flow responses across all four experimental tasks (Pearson $r=0.62, P<0.001$ ).

We observed activity relating to the voluntary intent to relax in the left anterior cingulate, globus pallidus and parietal cortex. In addition, we observed activation of the anterior cingulate and cerebellar vermis that was specifically associated with the influence of biofeedback on intentional relaxation, and right medial temporal lobe activity, adjacent to the amygdala, that was related to the recorded rate of sympathetic relaxation across all tasks. These functional neuroanatomical findings suggest differential regional contributions to the control of bodily states of sympathetic arousal.

Performance of relaxation tasks elicited maximal activation in the left anterior cingulate. This region of the limbic cortex has been implicated in cognitive and emotional processing and as part of a midline attentional system that involves the bilateral dorsolateral prefrontal and parietal lobe cortices (Nobre et al., 1997; Mesulam, 1999). Although relaxation involved a narrowing of the attentional focus to somatic responses (e.g. breathing), it is unlikely that attentional modulation provides the unique explanation for the cingulate activation we observed. Lesions to the anterior cingulate cortex may have a limited impact on global attention (e.g. Turken and Swick, 1999) but compromise autonomic responsivity, including sympathetic skin responses (Tranel and Damasio, 1994). Enhanced anterior cingulate activity has been related previously to autonomic arousal (e.g. task-independent increases in blood pressure) (Critchley et al., 2000a), in which a specific modulatory effect is seen when autonomic responses are absent as a result of peripheral autonomic denervation (Critchley et al., 2001).

Neuroimaging studies suggest not only an association between anterior cingulate activity and autonomic responses, but also the lateralization of this association. We observed predominantly left cingulate activity associated with the intention to relax, which contrasts with right cingulate activity 
observed during task-induced states of sympathetic arousal (Critchley et al., 2000a). We were also able to explore left anterior cingulate activity in relation to the measured rates of sympathetic relaxation achieved during scanning. Consistent with both the representation of intention to relax and modulation by feedback, left anterior cingulate activity discriminated between relaxation and non-relaxation tasks and decreased when relaxation was achieved. Thus, our evidence suggests that the left anterior cingulate is involved in the intentional modulation of bodily states of arousal, consistent with an executive behavioural role, and is itself modulated by afferent feedback from the periphery concerning the impact on bodily response of these intentions (Critchley et al., 2001).

In addition to the anterior cingulate cortex, the intention to relax was associated with increased activity in the globus pallidus and inferior parietal lobule. Although the behavioural act of relaxation necessarily involves a decrease in motor activity, this is unlikely to be the sole explanation for the observed increase in pallidal activity. Globus pallidus activity discriminated between relaxation and non-relaxation tasks, but was also modulated by breach of intention independently of whether the subject was trying to relax. Thus, correlation analyses showed significant increases in pallidal activity with decreases in sympathetic tone when subjects intended not to relax and also when subjects failed to relax during the relaxation tasks. This error-detection function is consistent with neurophysiological evidence implicating striatal activity in signalling errors in predicted outcomes (Schultz et al., 2000). However, the anterior cingulate (Carter et al., 1998; Kiehl et al., 2000) and orbitofrontal cortex (Iversen and Mishkin, 1970; Rolls et al., 1996; Nobre et al., 1999) may also contribute to the detection of mismatches between intention (or expectancy) with afferent feedback.

The contribution of the left inferior parietal lobule to the cognitive intention to achieve sympathetic relaxation is interesting, as enhanced activity in the right inferior parietal lobule activity has been associated with sympathetic arousal (Critchley et al., 2000b). The right inferior parietal lobule is strongly implicated in directing attention to external stimuli (e.g. Fink et al., 1996; Kastner et al., 1999), and our earlier findings suggest a common neural substrate for selective attention and arousal within the right parietal lobe (Critchley et al., 2000b). In this context, the present findings suggest the lateralization of parietal activity, cognitively driven modulation of sympathetic tone being associated with enhanced activity in the left parietal lobe and sympathetic arousal with activation in the right inferior parietal lobe. Thus, one interpretation of these findings is that they suggest that activity in the left inferior parietal lobule is related to attention to internal states rather than external stimuli.

Biofeedback relaxation is a useful experimental tool for eliciting decreases in sympathetic tone and may also have valuable therapeutic benefits in training symptomatic subjects strategies to decrease emotional arousal (e.g. Leahy et al., 1998). Activity relating to biofeedback relaxation, in addition to brain areas more directly associated with the intent to relax, was observed in the anterior cingulate cortices and cerebellar vermis. These brain areas have been implicated previously in cardiovascular arousal, independently of how that arousal is engendered (Critchley et al., 2000a), observations that implicate this region in the integration of bodily responses with cognitive intent. Surprisingly, we observed that greater right than left cingulate activity was unique to biofeedback relaxation.

We found an association between right medial temporal lobe activity, anterior and inferior to the amygdala, and the rate of autonomic relaxation. Activity in the amygdala region is strongly associated with autonomic responses, sympathetic arousal usually accompanying emotional states such as fear and threat (Reis and Ledoux, 1987; Furmark et al., 1997; Buchel et al., 1998). The present study identified medial temporal lobe activity, adjacent to the amygdala, that reflected relaxation of sympathetic tone independently of the intention to relax. Our methods do not allow us to determine if this activity reflects efferent control of sympathetic tone (mediated perhaps through amygdala connections with brainstem nuclei) or afferent representation of bodily relaxation. However, the relative task-independence of this medial temporal lobe activity suggests a close relationship with true physiological relaxation in a region more commonly associated with efferent signalling of changes in bodily state. More speculatively, amygdala activity has been implicated in stress responses, mood and anxiety disorders (Drevets et al., 1992; Morris et al., 1996; Birbaumer et al., 1998; Rauch et al., 2000). Our findings indicate that a region adjacent to the amygdala might serve directly in reducing the sympathetic bodily responses commonly associated with stress and anxiety.

Although personality factors and sex differences may exist in the responses of individuals to relaxation training and biofeedback (Davidson and Schwartz, 1976), our observations were limited to male subjects who were trained on the biofeedback relaxation task. Within this group, we examined the functional neuroanatomy related to cognitively mediated decreases in sympathetic relaxation when subjects performed a biofeedback relaxation exercise, a relaxation exercise without biofeedback and corresponding control (nonrelaxation) tasks. The findings provide further evidence for distinct neural mechanisms involved in the central control of autonomic bodily responses and their modulation by cognitive intent. A noticeable feature of the findings is that they highlight regions implicated in emotional processing and emotional disorders (Drevets et al., 1992; Morris et al., 1996; Birbaumer et al., 1998; Saxena et al., 1998; Chua et al., 1999; Rauch et al., 2000). An intriguing possibility raised by the findings is that the efficacy of psychological interventions may be mediated by modulation of activity in these regions.

\section{Acknowledgements}

This work was supported by a Wellcome Trust Programme Grant to R.J.D., a Wellcome Short-Term Travel Fellowship 
to R.N.M. and an award from the Paul Balint Trust during his sabbatical to the Institute of Neurology and a Clinical Research Fellowship to H.D.C. from the Brain Research Trust. We acknowledge the help and advice of O. Josephs and other members of the Wellcome Department of Cognitive Neurology.

\section{References}

Bechara A, Tranel D, Damasio H, Adolphs R, Rockland C, Damasio AR. Double dissociation of conditioning and declarative knowledge relative to the amygdala and hippocampus in humans. Science 1995; 269: 1115-8.

Bechara A, Tranel D, Damasio H, Damasio AR. Failure to respond autonomically to anticipated future outcomes following damage to prefrontal cortex. Cereb Cortex 1996; 6: 215-25.

Bechara A, Damasio H, Tranel D, Damasio AR. Deciding advantageously before knowing the advantageous strategy. Science 1997; 275: 1293-5.

Birbaumer N, Grodd W, Diedrich O, Klose U, Erb M, Lotze M, et al. fMRI reveals amygdala activation to human faces in social phobics. Neuroreport 1998; 9: 1223-6.

Boucsein W. Electrodermal activity. New York: Plenum Press; 1992.

Buchel C, Morris J, Dolan RJ, Friston KJ. Brain systems mediating aversive conditioning: an event-related fMRI study. Neuron 1998; 20: $947-57$.

Cahill L. The neurobiology of emotionally influenced memory. Implications for understanding traumatic memory. [Review]. Ann N Y Acad Sci 1997; 821: 238-46.

Carter CS, Braver TS, Barch DM, Botvinick MM, Noll D, Cohen JD. Anterior cingulate cortex, error detection, and the online monitoring of performance. Science 1998; 280: 747-9.

Chua P, Krams M, Toni I, Passingham R, Dolan RJ. A functional anatomy of anticipatory anxiety. Neuroimage 1999; 9: 563-71.

Critchley HD, Corfield DR, Chandler MP, Mathias CJ, Dolan RJ. Cerebral correlates of autonomic cardiovascular arousal: a functional neuroimaging investigation in humans. J Physiol (Lond); 2000a; 523: $259-70$.

Critchley HD, Elliott, R, Mathias CJ, Dolan RJ. Neural activity relating to generation and representation of galvanic skin conductance responses: a functional magnetic resonance imaging study. J Neurosci 2000b; 20: 3033-40.

Critchley HD, Mathias CJ, Dolan RJ. Neural correlates of first and second-order representation of bodily states. Nat Neurosci. 2001; 4: $207-12$

Damasio AR. Descartes' error. Emotion, reason, and the human brain. New York: Putnam; 1994.

Damasio AR, Tranel D, Damasio HC. Somatic markers and the guidance of behavior: theory and preliminary testing. In: Levin HS, Eisenberg HM, Benton AL, editors. Frontal lobe function and dysfunction. New York: Oxford University Press, 1991. p. 217-29.

Davidson RJ, Schwartz GE. Patterns of cerebral lateralization during cardiac biofeedback versus the self-regulation of emotion: sex differences. Psychophysiology 1976; 13: 62-8.

Dawson ME, Schell AM, Filion DL. The electrodermal system. In: Cacioppo JT, Tassinary LG, Berntson GG, editors. Handbook of psychophysiology. 2nd ed. Cambridge: Cambridge University Press; 2000. p. 200-23.

Drevets WC, Videen TO, Price JL, Preskorn SH, Carmichael ST, Raichle ME. A functional anatomical study of unipolar depression. J Neurosci 1992; 12: 3628-41.

Duvernoy HM. The human brain: surface, three-dimensional sectional anatomy and MRI. Wien: Springer-Verlag; 1991

Fink GR, Halligan PW, Marshall JC, Frith CD, Frackowiak RS Dolan RJ. Where in the brain does visual attention select the forest and the trees? Nature 1996; 382: 626-8.

Fish DR, Gloor P, Quesney FL, Olivier A. Clinical responses to electrical brain stimulation of the temporal and frontal lobes in patients with epilepsy. Pathophysiological implications. Brain 1993; 116: $397-414$.

Fowles DC, Christie MJ, Edelberg R, Grings WW, Lykken DT, Venables PH. Publication recommendations for electrodermal measurements. Psychophysiology 1981; 18: 232-9.

Fredrikson M, Furmark T, Olsson MT, Fischer H, Andersson J, Langstrom B. Functional neuroanatomical correlates of electrodermal activity: a positron emission tomographic study. Psychophysiology 1998; 35: 179-85.

Friston K, Ashburner J, Frith CD, Poline J-B, Heather JD, Frackowiak RSJ. Spatial registration and normalization of images. Hum Brain Mapp 1995a; 3: 165-89.

Friston KJ, Holmes AP, Worsley KJ, Poline J-B, Frith CD, Frackowiak RSJ. Statistical parametric maps in functional imaging: a general linear approach. Hum Brain Mapp 1995b; 2: 189-210.

Furmark T, Fischer H, Wik G, Larsson M, Fredrikson M. The amygdala and individual differences in human fear conditioning. Neuroreport 1997; 8: 3957-60.

Iversen SD, Mishkin M. Perseverative interference in monkeys following selective lesions of the inferior prefrontal convexity. Exp Brain Res 1970; 11: 376-86.

Kastner S, Pinsk MA, De Weerd P, Desimone R, Ungerleider LG. Increased activity in human visual cortex during directed attention in the absence of visual stimulation. Neuron 1999; 22: 751-61.

Kiehl KA, Liddle PF, Hopfinger JB. Error processing and the rostral anterior cingulate: an event-related fMRI study. Psychophysiology 2000; 37: 216-23.

Lazar SW, Bush G, Gollub RL, Fricchione GL, Khalsa G, Benson $H$. Functional brain mapping of the relaxation responses and meditation. Neuroreport 2000; 11: 1581-5.

Leahy A, Clayman C, Mason I, Lloyd G, Epstein O. Computerised biofeedback games: a new method for teaching stress management and its use in irritable bowel syndrome. J R Coll Physicians Lond 1998; 32: 552-6.

Mesulam MM. Spatial attention and neglect: parietal, frontal and cingulate contributions to the mental representation and attentional 
targeting of salient extrapersonal events. [Review]. Philos Trans Roy Soc Lond B Biol Sci 1999; 354: 1325-46.

Morris JS, Frith CD, Perrett DI, Rowland D, Young AW, Calder AJ, et al. A differential neural response in the human amygdala to fearful and happy facial expressions. Nature 1996; 383: 812-5.

Nobre AC, Sebestyen GN, Gitelman DR, Mesulam MM, Frackowiak RS, Frith CD. Functional localization of the system for visuospatial attention using positron emission tomography. Brain 1997; 120: 515-33.

Nobre AC, Coull JT, Frith CD, Mesulam MM. Orbitofrontal cortex is activated during breaches of expectation in tasks of visual attention. Nat Neurosci 1999; 2: 11-12.

Oppenheimer SM, Gelb A, Girvin JP, Hachinski VC. Cardiovascular effects of human insular cortex stimulation. Neurology 1992; 42 1727-32.

Pool JL, Ransohoff J. Autonomic effects on stimulating the rostral portion of the cingulate gyri in man. J Neurophysiol 1949; 12 : 385-92.

Rainville P, Hofbauer RK, Paus T, Duncan GH, Bushnell MC, Price DD. Cerebral mechanisms of hypnotic induction and suggestion. J Cogn Neurosci 1999; 11: 110-25.

Rauch SL, Whalen PJ, Shin LM, McInerny SC, Macklin ML, Lasko NB, et al. Exaggerated amygdala response to masked facial stimuli in posttraumatic stress disorder: a functional MRI study. Biol Psychiatry 2000; 47: 769-76.

Reis DJ, Ledoux JE. Some central neural mechanisms governing resting and behaviourally coupled control of blood pressure. [Review]. Circulation 1987; 76: I2-9.

Rolls ET, Critchley HD, Mason R, Wakeman EA. Orbitofrontal cortex neurons: role in olfactory and visual association learning. J Neurophysiol 1996; 75: 1970-81.

Russek LG, King SH, Russek SJ, Russek HI. The Harvard Mastery of Stress Study 35-year follow-up: prognostic significance of patterns of psychophysiological arousal and adaptation. Psychosom Med 1990; 52: 271-85.

Saxena S, Brody AL, Schwartz JM, Baxter LR. Neuroimaging and fronto-subcortical circuitry in obsessive-compulsive disorder. [Review]. Br J Psychiatry Suppl 1998; 35: 26-37.

Schultz W, Tremblay L, Hollerman JR. Reward processing in primate orbitofrontal cortex and basal ganglia. Cereb Cortex 2000; 10: $272-84$

Soufer R, Bremner JD, Arrighi JA, Cohen I, Zaret BL, Burg MM, et al. Cerebral cortical hyperactivation in response to mental stress in patients with coronary artery disease. Proc Natl Acad Sci USA 1998; 95: 6454-9.

Steptoe A, Cropley M, Joekes K. Job strain, blood pressure and response to uncontrollable stress. J Hypertens 1999; 17: 193-200.

Talairach J, Tournoux P. Co-planar stereotaxic atlas of the human brain. Stuttgart: Thieme; 1988.

Tranel D, Damasio H. Neuroanatomical correlates of electrodermal skin conductance responses. Psychophysiology 1994; 31: 427-38.

Turken AU, Swick D. Response selection in the human anterior cingulate cortex. Nat Neurosci 1999; 2: 920-4.

Venables PH, Christie MJ. Electrodermal activity. In: Martin I, Venables PH, editors, Techniques in psychophysiology. Chichester: John Wiley; 1980. p. 3-67.

Williamson JW, Nobrega AC, McColl R, Mathews D, Winchester $\mathrm{P}$, Friberg L, et al. Activation of the insular cortex during dynamic exercise in humans. J Physiol (Lond) 1997; 503: 277-83.

Worsley KJ, Marrett S, Neelin P, Vandal AC, Friston KJ, Evans AC. A unified statistical approach for determining significant signals in images of cerebral activation. Hum Brain Mapp 1996; 4: $58-73$.

Received October 5, 2000. Revised December 1, 2000. Accepted January 15, 2001 